\title{
EVALUACIÓN DE LA ANSIEDAD EN ESTUDIANTES DE LA CARRERA DE Cirujano Dentista
}

\author{
Laura Edna Aragón Borja \\ Margarita Chávez Becerra \\ María de los Ángeles Flores Tapia \\ UNAM FES IZTACALA
}

MÉXICO

\begin{abstract}
RESUMEN
Debido a que la ansiedad es una conducta que se presenta con bastante frecuencia entre la población de estudiantes universitarios y repercute negativamente en su aprendizaje, el objetivo de este estudio fue evaluar la ansiedad de estudiantes de la Carrera de Cirujano Dentista de la FES Iztacala UNAM, para lo cual se aplicó a una muestra aleatoria de 363 estudiantes el Inventario de Situaciones y Respuestas de Ansiedad de Tobal y Cano (1997). Los resultados muestran que entre el $12 \%$ y $19 \%$ de los estudiantes están presentando algún tipo de ansiedad. Al comparar la ansiedad entre hombres y mujeres, se encontraron diferencias significativas a favor de las mujeres para las modalidades cognitiva, fisiológica y total de ansiedad. Con respecto al grado escolar, sólo existen diferencias significativas en la modalidad de ansiedad cognitiva, siendo los más ansiosos los estudiantes de séptimo semestre con respecto a los de primero y quinto, y los de tercer semestre con respecto a los de quinto. Se realizaron también otras comparaciones utilizando la d de Cohen, encontrándose resultados interesantes en ansiedad fisiológica y motora. Se discuten los resultados relacionándolos con las demandas escolares de los estudiantes de esta carrera.
\end{abstract}

Palabras Clave: Ansiedad, ansiedad cognitiva, fisiológica y motora, Cirujano Dentista

\section{AnXIETy EVALUATION In Dental SURgEON StUdent}

\section{ABSTRACT}

Due to anxiety is a behavior that arises quite frequently among the population of college students and negatively impact on their learning, the aim of this study was to evaluate anxiety in a random sample of 363 students from first to seventh semesters of Dental Surgeon Career of FES Iztacala UNAM, using for this purpose the Situaciones y Respuestas de Ansiedad Inventory (Tobal \& Cano, 1997). Results showed that between $12 \%$ and $19 \%$ of students are presenting some kind of anxiety. Comparing sex, significant differences in favor of women were found at cognitive, physiological and total modalities of anxiety. With regard to the scholar grade, there are significant differences only in cognitive anxiety, being the most anxious seventh semester students compared with the first and the fifth, and the third semester students compared to the fifth. Using other comparisons with Cohen'd, it was found interesting results in physiological and motor anxiety. Results are discussed in relation of the school demands of the students of this Career.

Keywords: Anxiety; cognitive, physiological and motor anxiety, Dental Surgeon

Aragón, B. L. E., Chávez, B. M. \& Flores, T. M. A. (2015). Evaluación de la ansiedad en estudiantes de la carrera de cirujano dentista. Revista Digital Internacional de Psicología y Ciencia Social, 1(1), pp. 33-42. 


\section{BITÁCORA DEL ARTí́CULO}

\section{EVALUACIÓN DE LA ANSIEDAD EN ESTUDIANTES DE LA CARRERA DE CIRUJANO DENTISTA Laura Edna Aragón Borja, Margarita Chávez Becerra y María de los Ángeles Flores Tapia}

\section{Bitácora del Artículo:}

Recibido: 10 de febrero de 2014

Aceptado: 6 de noviembre de 2014

Publicado en línea: 7 de febrero de 2015

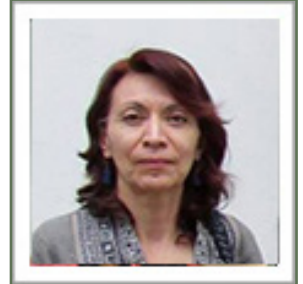

Laura Edna Aragón

UNAM - FES Iztacala

Correo: aragonb@gmail.com

Profesora Titular "B". Licenciatura en Psicología, Especialidad Matemáticas Aplicadas a Psicología y Maestría en Modificación de Conducta. Directora de la Unidad de Evaluación Psicológica Iztacala (UEPI). Autora de varios libros sobre evaluación.

Ver más..

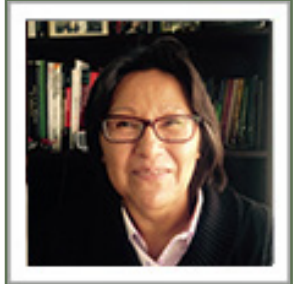

Margarita Chávez Becerra

UNAM - FES Iztacala

Correo: margaritachabe@gmail.com

Profesora Titular "A". Con grado de Maestría en Modificación de Conducta por la FES-Iztacala, UNAM. Impartiendo la asignatura curricular de Métodos Cuantitativos en la carrera de psicología, FES Iztacala, UNAM, por más de 25 años.

Ver más...

\section{Contribución de las autoras}

La primera autora, Laura Edna Aragón Borja concibió el estudio y participó en todos los rubros del artículo | Margarita Chávez Becerra en los rubros de Método, Análisis de datos, conclusiones y discusión | Ma. de los Ángeles Flores Tapia, en las conclusiones y discusión | Las tres autoras en la revisión del manuscrito.

\section{Agradecimientos}

Agradecimiento especial a la carrera de odontología de la UNAM, FES-Iztacala.

\section{Datos de filiación de las autoras}

Laura Edna Aragón Borja | Carrera de psicología y maestría en terapia familiar. UNAM, FES-Iztacala | Margarita Chávez Becerra | Carrera de psicología. UNAM, FES-Iztacala | María de los Ángeles Flores Tapia | Carrera de odontología. UNAM, FES-Iztacala | 


\section{TABLA DE CONTENIDO}

INTRODUCCIÓN

MÉTOdo

Diseño, 37

Muestra, 37

Instrumento, 38

Procedimiento, 38

RESULTADOS

CONCLUSIONES

REFERENCIAS

MetA-AnÁlisis del Artículo 43

Dimensión Cuantitativa, 44

Dimensión Cualitativa, 46

Ir a la Historia del Proceso Editorial 
Introducción

\begin{abstract}
I a ansiedad es un repertorio que suele estar presente con bastante frecuencia entre la población de estudiantes universitarios; Sue, Sue y Sue (2000) consideran que estar vivos significa que estamos constantemente expuestos a estresores tales como problemas sociales, enfermedades, búsqueda de empleo y desde luego, las exigencias escolares. Cuando los estudiantes son confrontados con demandas externas excesivas, sus conductas de afrontamiento pueden fallar $y$, al no tener los recursos apropiados y usar estrategias inadecuadas para encararlas, el resultado puede ser la aparición de síntomas psicofisiológicos, apatía, ansiedad, pánico, depresión, violencia, entre otros. Así, es importante detectar oportunamente la ansiedad, ya que es una conducta inadaptativa que puede interferir con el buen curso del desempeño escolar de los estudiantes.
\end{abstract}

Algunos estresores que se les presentan a los estudiantes universitarios y que les pueden provocar ansiedad son: la participación y las exposiciones en clase, trabajar en equipo, la elaboración de tareas, las evaluaciones y exámenes, el tiempo limitado que se posee para la realización de las actividades académicas $y$, en algunas carreras, la atención a los pacientes.

La ansiedad puede ser producida tanto por estresores externos o situacionales, percibidos por el sujeto como peligrosos o amenazantes, como por estímulos internos como los pensamientos. Se conceptualiza a la ansiedad como: “...la respuesta, o patrón de respuestas, que engloba aspectos cognitivos displacenteros, de tensión y aprensión; aspectos fisiológicos, caracterizados por un alto grado de activación del sistema nervioso autónomo y aspectos motores que suelen implicar comportamientos poco ajustados y escasamente adaptativos" (Tobal \& Cano, 1997, p. 9).

La ansiedad interfiere tanto con el bienestar emocional como con el desempeño académico; entre mayor sea el nivel de ansiedad experimentado por una persona, más altas son las probabilidades de que sus respuestas fisiológicas, cognitivas o motoras interfieran con el desempeño que el individuo tenga al momento de realizar diversas actividades que requieran de procesos cognitivos superiores. En las actividades llevadas a cabo para el aprendizaje y el estudio se encuentran involucrados procesos cognitivos superiores (atención, concentración, memoria a corto y largo plazo, percepción, procesamiento de la información, entre otros); si los efectos de la ansiedad perturban dichos procesos, entonces la adquisición de conocimientos y el aprendizaje se verán disminuidos. Es importante considerar esto debido a que las presiones a las que se ven sometidos los estudiantes pueden interferir con la calidad de aprendizaje y por lo tanto la adquisición de conocimientos se puede ver seriamente afectada. En los estudiantes universitarios, principalmente en aquellos de las carreras del área de la salud, es de suma importancia que la adquisición de conocimientos sea adecuada, ya que si se presentan fallas en el aprendizaje debido a la presencia de ansiedad, esto puede repercutir negativamente en la calidad de los servicios prestados a los usuarios.

En un estudio llevado a cabo por García y de la Peza (2005), el objetivo era determinar la correlación entre el rendimiento académico total y por materias, medido a través de las calificaciones escolares de los alumnos y la ansiedad medida a través del Inventario de Situaciones y Respuestas de Ansiedad (ISRA) de Tobal y Cano, en una muestra de 151 alumnos del Instituto Tecnológico y de Estudios Superiores de Monterrey Campus Querétaro; se encontró una correlación significativa negativa entre el desempeño académico y la ansiedad; esto es, aquellos alumnos que presentaron las puntuaciones más altas en ansiedad, presentaron a su vez menor rendimiento académico que aquellos con puntuaciones bajas en ansiedad.

Por su parte, Sandin, Valiente, Chorot y Santed (2005) realizaron un estudio con una muestra de 726 estudiantes de 18 a 34 años de edad, de las universidades de Madrid y Pamplona, a quienes se les aplicaron dos cuestionarios de ansiedad, encontrando puntuaciones significativamente más elevadas en las mujeres en comparación con los hombres en las puntuaciones totales del índice de ansiedad así como en las escalas somática y cognitiva, pero no así en la escala social; asimismo, encontraron correlaciones significativas entre las escalas de ansiedad total, cognitiva, somática y social, con los miedos, el afecto negativo, el neuroticismo y la depresión.

En otro estudio de Sánchez, Aparicio y Dresch (2006), el objetivo era analizar las relaciones entre algunas variables psicológicas que influyen en la salud (ansiedad, autoestima y satisfacción autopercibida) y 
determinar las diferencias entre hombres y mujeres. La muestra fue de 500 sujetos de 25 a 65 años seleccionados aleatoriamente de la ciudad de Madrid. No se encontraron diferencias según el género en las variables enfermedades crónicas, ansiedad motora, autoestima y satisfacción vital. Las diferencias significativas aparecen en visitas al médico, dolencias físicas, ansiedad cognitiva, ansiedad fisiológica y ansiedad total, en las que las mujeres puntúan más, y en salud física percibida, donde los hombres puntúan más. La varianza explicada por las variables psicológicas respecto a los índices de salud física, en el caso de las mujeres en comparación con los hombres, está concentrada sobre todo en ansiedad fisiológica. Concluyen los autores que la salud física de las mujeres, más que la de los varones, está íntimamente relacionada con la salud psicológica.

Por su parte, Hernández-Pozo, Coronado, Araujo y Cerezo (2008) llevaron a cabo un estudio con 22 alumnos mexicanos de 20 a 26 años, de sexto semestre de una universidad pública, para explorar la relación entre el rendimiento académico y dos medidas de ansiedad, conductual y fisiológica, obtenidas respectivamente a través de una tarea computacional mediante el paradigma de Stroop y registros de la presión arterial y el pulso. Los resultados mostraron que los alumnos con más bajos índices de aprovechamiento académico exhibían significativamente mayores índices de respuestas de ansiedad, tanto conductual como fisiológica. En una réplica de este estudio con 81 estudiantes del primer semestre de la carrera de Medicina se encontraron resultados semejantes, esto es, los estudiantes cuyo nivel de ansiedad era bajo o que no mostraron ansiedad obtenían mejores promedios en su desempeño escolar que aquellos que presentaban ansiedad (Torres, Hernández-Pozo, Castillo, Coronado y Cerezo, 2008).

En otra investigación llevada a cabo por Aragón, Contreras y Tron (2011), se aplicó el ISRA a una muestra aleatoria de 149 estudiantes universitarios con edades comprendidas entre los 17 y 28 años, de los semestres $1^{\circ}$ al $9^{\circ}$, de las carreras de Ciencias Políticas, Ingeniería Agrícola, Ingeniería en Computación, Ingeniería Química, Médico Cirujano, Químico Farmacobiólogo, Relaciones Internacionales y Veterinaria. Se encontró que $12 \%$ de los alumnos presentaron ansiedad cognitiva, $15.5 \%$ ansiedad fisiológica y $15 \%$ ansiedad motora. En las tres modalidades de ansiedad, las mujeres sistemáticamente obtuvieron puntuaciones más altas que los hombres; sin embargo, al realizar un análisis con la $t$ de Student para comparar la ejecución de hombres y mujeres, se observó que sólo fue significativa la diferencia en ansiedad fisiológica, esto es, ante acontecimientos que las estresan, las mujeres presentan, en comparación con los hombres, más respuestas fisiológicas.

Finalmente, en otro estudio cuyo objetivo era obtener el perfil de personalidad, se trabajó con una muestra de 345 estudiantes universitarios de la carrera de Cirujano Dentista de la FES Iztacala (Aragón y Flores, 2014), a quienes se les aplicó el Cuestionario 16 PF de Cattell. En esta investigación presentaron rasgos de ansiedad las mujeres en $3^{\circ}, 5^{\circ}$ y $7^{\circ}$ semestres y los hombres en $3^{\circ}$ y $5^{\circ}$ semestres. De hecho, estos resultados fueron los que motivaron a explorar la ansiedad que presentan los estudiantes universitarios de dicha carrera en la Facultad de Estudios Superiores Iztacala (FES Iztacala); se decidió entonces aplicar un instrumento que específicamente midiera ansiedad en una muestra aleatoria de estudiantes de la Carrera de Cirujano Dentista, con la peculiaridad además de hacerlo al inicio del ciclo escolar (en la segunda semana de haber iniciado el semestre escolar), para eliminar sesgos por las demandas escolares en etapas intermedias o avanzadas del semestre.

\section{Método}

\section{Diseño}

Teniendo en cuenta que el interés del estudio se centra en la evaluación de la ansiedad, el diseño es no experimental, descriptivo y transversal.

\section{Muestra}

La muestra de este estudio fueron 363 estudiantes de la Carrera de Cirujano Dentista de la FES Iztacala, 233 mujeres (64\%) y 130 hombres (36\%), con edades comprendidas entre 17 y 31 años $(M=20$ años, $D E=$ 3.38), concentrándose el $88 \%$ entre 18 y 22 años. Se eligió este tamaño de muestra, aproximadamente el $15 \%$ de la población total, con la finalidad de poder generalizar los resultados a la población total. La muestra fue elegida aleatoriamente seleccionando al azar a tres grupos por semestre lectivo, quedando conformada así por 88 alumnos del primer semestre, 91 del tercero, 102 del quinto y 82 del séptimo semestre. La Tabla 1 muestra la composición de la muestra por sexo y semestre cursado. 
Tabla 1

Composición de la muestra del estudio por sexo y semestre

\begin{tabular}{|cccccc|}
\hline \multicolumn{4}{c}{ Mujeres } & \multicolumn{2}{c}{ Hombres } \\
Semestre & Frecuencia & Porcentaje & Frecuencia & Porcentaje & \% por Semestre \\
\hline $\mathbf{1}^{\circ}$ & 56 & 64 & 32 & 36 & 24 \\
\hline $\mathbf{3}^{\circ}$ & 58 & 64 & 33 & 36 & 25 \\
$\mathbf{5}^{\circ}$ & 68 & 67 & 34 & 33 & 28 \\
\hline $\mathbf{7}^{\circ}$ & 51 & 62 & 31 & 38 & 23 \\
\hline TOTAL & 233 & 64 & 130 & 36 & 100 \\
\hline
\end{tabular}

\section{Instrumento}

Se utilizó el Inventario de Situaciones y Respuestas de Ansiedad (ISRA) de Tobal y Cano (1997). Éste es un cuestionario que mide ansiedad a través de 66 reactivos tipo Likert repartidos en tres escalas de ansiedad: cognitiva, fisiológica y motora, que se cruzan con 23 situaciones categorizadas en cuatro diferentes tipos: situaciones relacionadas con evaluación y asunción de responsabilidades, situaciones que implican relaciones interpersonales y sexuales, situaciones de tipo fóbico y situaciones habituales de la vida cotidiana; ante cada una de ellas, el sujeto tiene que ponderarlas y anotar con qué frecuencia le ocurre esa combinación situación-respuesta: casi nunca, pocas veces, unas veces sí y otras veces no, muchas veces o casi siempre; a mayor frecuencia de ocurrencia mayor puntuación, lo que indicaría mayor intensidad de la ansiedad medida. Este instrumento nos proporciona así cuatro medidas: ansiedad cognitiva (sentimientos de terror, amenaza o catástrofe inminente, pensamientos e imágenes altamente específicos relacionados con miedo); ansiedad fisiológica (cambios cardiovasculares, aumento de la actividad electrodérmica, del tono músculo esquelético y del ritmo respiratorio); ansiedad motora (inquietud, tics, temblores, gesticulación, tartamudeo, lengua trabada; reducción de la destreza motora, de la habilidad para la discriminación perceptiva, del tiempo de reacción y de la ejecución de tareas complejas; conductas de evitación y escape) y ansiedad total (una puntuación global que abarca a las tres anteriores).

Se eligió este instrumento por las siguientes ventajas: se desarrolló originalmente para población hispana, posee una alta consistencia interna (alfa de Cronbach $=0.99$ ), pero sobre todo, porque no conceptualiza a la ansiedad como un constructo unitario, sino que considera que las respuestas de sistemas distintos en el individuo no siempre varían en la misma forma, por lo que evalúa por separado la frecuencia de respuestas cognitivas, fisiológicas y motoras de ansiedad, ante situaciones susceptibles de generarla; además, es una prueba objetiva, donde, a decir de los autores, tanto las situaciones como las conductas aparecen descritas en forma precisa y específica, es decir, se describen situaciones y conductas concretas y no abstracciones de las mismas.

\section{Procedimiento}

Una vez aleatorizados los grupos que participarían en el estudio, se solicitó permiso a los profesores de dichos grupos para aplicarles el ISRA en sus horas de clase y se les pidió a los alumnos su consentimiento para cumplimentar el cuestionario. La aplicación para toda la muestra se llevó a cabo en la segunda semana de haber iniciado el semestre escolar, para eliminar los efectos debidos a las demandas de trabajo escolar que pudieran influir en los resultados.

La aplicación se realizó en los salones donde los alumnos tomaban clases, se les dieron las instrucciones oralmente y se les instruyó a contestar sinceramente el cuestionario, asegurándoles que se guardaría en el anonimato sus resultados ya que los únicos datos que tendrían que poner en el cuestionario eran el sexo, semestre, turno y edad. La captura de los datos, así como los análisis se realizaron mediante el paquete estadístico para las ciencias sociales (SPSS), versión 20.

\section{Resultados}

De los 363 estudiantes a los que se les aplicó el inventario, presentaron ansiedad total 14\%; desglosando en las diferentes modalidades de ansiedad que el instrumento mide, $12 \%$ del total de la muestra presentaron ansiedad cognitiva, $19 \%$ ansiedad fisiológica y $14 \%$ ansiedad motora; como observamos, la modalidad más presente es la fisiológica (Ver Figura 1). 


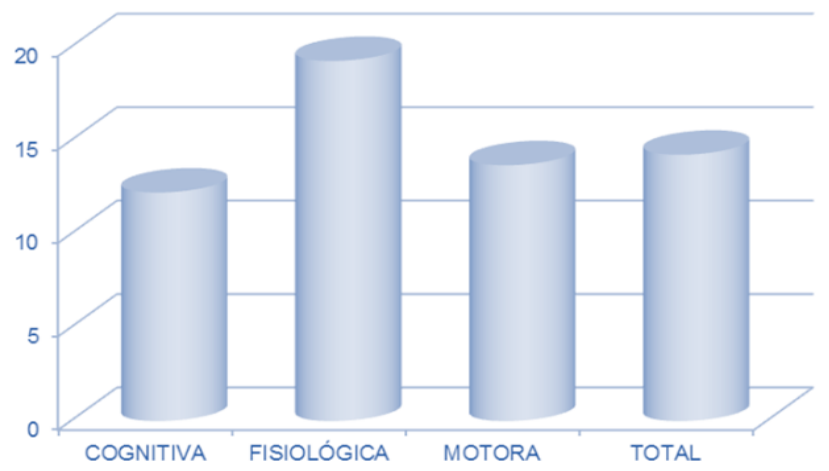

Figura 1.

Porcentaje de estudiantes en el total de ansiedad y en las modalidades evaluadas

Al comparar las medias de los puntajes $(M)$ que obtienen hombres y mujeres en las tres modalidades evaluadas de ansiedad y en la total, se puede observar (Ver Tabla 2), que las medias de los puntajes son mayores para las mujeres; sin embargo, al realizar una prueba $t$ de Student, las diferencias sólo resultan estadísticamente significativas para las modalidades cognitiva, fisiológica y el total de ansiedad en los tres casos a favor de las mujeres, esto es, las mujeres tienden en mayor medida que los hombres a manifestar sentimientos de amenaza, catástrofe o pensamientos relacionados con miedo (ansiedad cognitiva), así como cambios en el ritmo cardiovascular o respiratorio (ansiedad fisiológica), entre otros. Al realizar un análisis posterior acerca del tamaño del efecto utilizando la $d$ de Cohen, el cual se presenta en la Tabla 2, observamos que la puntuación promedio $(M=54.5)$ de las mujeres supera al $64.06 \%$ de los hombres en la modalidad de ansiedad cognitiva, mientras que en ansiedad fisiológica la puntuación promedio de las mujeres $(M=32.1)$, rebasa al $68.79 \%$ del grupo de los hombres; y en ansiedad motora las mujeres $(M=29.6)$ están por arriba de los hombres un $51.99 \%$. Para el total de ansiedad, la puntuación promedio de las mujeres $(M=116)$ supera al $61.41 \%$ de los hombres. Conforme a los resultados del tamaño del efecto (d) (González, 2014), se complementa la información proporcionada con la prueba t de Student.

Por otra parte y con la finalidad de evidenciar si los niveles de ansiedad total y de las modalidades cognitiva, fisiológica y motora, evaluadas por el ISRA, difieren o son explicados por el semestre que se cursa $\left(1^{\circ}, 3^{\circ}, 5^{\circ}\right.$ y $\left.7^{\circ}\right)$, se aplicó el análisis de varianza (ANOVA) de una entrada, resultando que sólo existen diferencias significativas en la modalidad de ansiedad cognitiva, es decir, la presencia de pensamientos asociados al miedo y sentimientos de amenaza o catástrofe entre los universitarios son disímiles entre los semestres que se cursan $\left(F_{(3,359)}=3.36, p=.019\right)$; en las otras modalidades de ansiedad las diferencias de medias no fueron significativas. Al efectuar contrastaciones por pares de medias a posteriori del ANOVA para la modalidad de ansiedad cognitiva a través de la prueba de diferencias mínimas significativas (DMS), se identifican diferencias significativas entre los estudiantes que cursan primer semestre $(M=46.8, D E=27.9)$ y los de séptimo $(M=$ 57.2, $\mathrm{DE}=27.9), d=.36, I C$ 95\%[.06,.67]; entre los de tercer semestre $(M=54.4, D E=32.7)$ y los de quinto $(M$ $=45.9, \mathrm{DE}=27.5), d=.29, I C 95 \%[.02, .58] ; \mathrm{y}$ entre los de séptimo semestre $(M=57.2, D E=27.9)$ y los de quinto $(M=45.9, \mathrm{DE}=27.5), d=.39, I C 95 \%[.11, .71]$.

En concordancia con los tamaños del efecto estimados para cada par de semestres contrastados, se tiene que el promedio de los puntajes de ansiedad cognitiva para los estudiantes que cursan séptimo semestre supera en un $64 \%$ al promedio de los estudiantes que

Tabla 2

Medidas descriptivas, valores $t$ de Student y probabilidades asociadas, tamaño del efecto (d) e intervalo de confianza del tamaño del efecto (IC 95\%), para la comparación del total de ansiedad y modalidades entre hombres y mujeres

\begin{tabular}{|l|c|c|c|c|c|c|c|c|c|c|}
\hline & \multicolumn{9}{c}{ Mujeres } & \multicolumn{9}{c|}{ Hombres } \\
\hline Ansiedad & $M$ & $n$ & DE & $M$ & $n$ & $D E$ & $t$ & $p$ & $d$ & $\begin{array}{l}\text { IC } \\
95 \%\end{array}$ \\
\hline Cognitiva & 54.5 & 233 & 29.42 & 44.2 & 130 & 27.26 & 3.3 & .00 & .36 & $\begin{array}{l}.29- \\
.43\end{array}$ \\
\hline Fisiológica & 32.1 & 232 & 14.60 & 23.1 & 126 & 20.89 & 3.5 & .00 & .49 & $.41-$ \\
\hline Motora & 29.6 & 233 & 25.56 & 28.2 & 130 & 26.17 & .49 & .62 & .05 & $.22-$ \\
& & & & & & & & & .36 \\
\hline Total & 116 & 232 & 73.24 & 95 & 124 & 67.93 & 2.7 & .01 & .29 & $.22-$ \\
& & & & & & & & & & .36 \\
\hline
\end{tabular}


cursan primer semestre; que el promedio del grupo de estudiantes de tercer semestre rebasa en $61.4 \%$ a los que cursan quinto semestre, así como que el promedio de los que cursan séptimo semestre está por arriba del $65 \%$ de los de quinto. Con esta información es evidente que el promedio de ansiedad cognitiva de los estudiantes de séptimo semestre es mayor que la que presentan los de primero y quinto semestres, y a su vez los estudiantes de tercer semestre ostentan más ansiedad cognitiva que los que cursan quinto semestre.

A pesar de que los resultados de los ANOVAs realizados al comparar los puntajes de ansiedad no resultaron significativos para ansiedad fisiológica $\left(F_{(3,354)}=1.91\right.$; $\mathrm{p}=.13)$, motora $\left(\mathrm{F}_{(3,359)}=1.75 ; \mathrm{p}=.16\right)$ y para ansiedad total $\left(F_{(3,352)}=2.40 ; p=.07\right)$; al estimar los tamaños del efecto ( $d$ de Cohen) para las comparaciones por pares entre semestres de cada una de las modalidades de ansiedad, se obtienen tamaños de efecto $(d)$ de muy pequeños hacia moderados, como se puede apreciar en la Tabla 3, por lo que las diferencias entre los pares por semestres para ansiedad fisiológica, motora y total, presentan magnitudes de consideración, por ejemplo, en el caso de ansiedad fisiológica entre los estudiantes de primer y quinto semestres $(d=.03)$, la puntuación promedio de los de primer semestre $(M=26.71)$ supera al $51.2 \%$ de los de quinto semestre. Para el par de séptimo y quinto semestres, también de ansiedad fisiológica, la puntuación promedio de los de séptimo semestre $(M=30.17)$ rebasa al $56.7 \%$ de los de quinto semestre. Para los pares de ansiedad motora entre los de tercer semestre y los de quinto, la puntuación promedio de los de tercero $(M=34)$ está por arriba de los de quinto en un $61.7 \%$. Respecto a los semestres séptimo y quinto del total de ansiedad, la puntuación promedio de los de séptimo semestre $(M=116.63)$ rebasa al $61 \%$ a los de quinto.

A pesar de que los resultados de los ANOVAs realizados al comparar los puntajes de ansiedad no resultaron significativos para ansiedad fisiológica $\left(F_{(3,354)}=1.91\right.$; $\mathrm{p}=.13)$, motora $\left(\mathrm{F}_{(3,359)}=1.75 ; \mathrm{p}=.16\right)$ y para ansiedad total $\left(F_{(3,352)}=2.40 ; p=.07\right)$; al estimar los tamaños del efecto ( $d$ de Cohen) para las comparaciones por pares entre semestres de cada una de las modalidades de ansiedad, se obtienen tamaños de efecto pequeños (.20 a .26), tendientes a moderados (.28 a .31) y no relevantes $(<.20)$, como se puede apreciar en la Tabla 3. De esta manera y sólo conforme a los tamaños de efecto pequeños y tendientes a moderados, destacan las diferencias entre los pares de semestres contrastados en ansiedad fisiológica, entre tercer y primer semestres $(d=.28)$, así como entre tercer y quinto semestres $(d=.29)$. Para ansiedad motora, las magnitudes entre
Tabla 3

Comparaciones pareadas por pares de semestres, medidas descriptivas, tamaños del efecto y porcentajes vinculados al tamaño del efecto

\begin{tabular}{|c|c|c|c|c|c|}
\hline Ansiedad & Semestres & Medias & DE. & d & $\%$ \\
\hline \multirow{10}{*}{ Fisiológica } & Primero & 26.71 & 19.63 & \multirow[t]{2}{*}{.03} & \multirow[t]{2}{*}{51.2} \\
\hline & Quinto & 26.01 & 20.89 & & \\
\hline & Tercero & 33.36 & 29.09 & \multirow[t]{2}{*}{.28} & \multirow[t]{2}{*}{61.0} \\
\hline & Primero & 26.71 & 19.63 & & \\
\hline & Tercero & 33.36 & 28.09 & \multirow[t]{2}{*}{.29} & \multirow[t]{2}{*}{61.4} \\
\hline & Quinto & 26.01 & 20.89 & & \\
\hline & Séptimo & 30.17 & 25.17 & \multirow[t]{2}{*}{.15} & \multirow[t]{2}{*}{55.9} \\
\hline & Primero & 26.71 & 19.63 & & \\
\hline & Séptimo & 30.17 & 25.17 & \multirow[t]{2}{*}{.17} & \multirow[t]{2}{*}{56.7} \\
\hline & Quinto & 26.01 & 20.89 & & \\
\hline \multirow{12}{*}{ Motora } & Primero & 28.17 & 25.51 & \multirow[t]{2}{*}{.10} & \multirow[t]{2}{*}{53.9} \\
\hline & Quinto & 25.66 & 23.05 & & \\
\hline & Tercero & 34.00 & 31.65 & \multirow[b]{2}{*}{.20} & \multirow[b]{2}{*}{50.7} \\
\hline & Primero & 28.17 & 25.51 & & \\
\hline & Tercero & 34.00 & 31.65 & \multirow[b]{2}{*}{.30} & \multirow[b]{2}{*}{61.7} \\
\hline & Quinto & 25.66 & 23.05 & & \\
\hline & Tercero & 34.00 & 31.65 & \multirow[t]{2}{*}{.31} & \multirow[t]{2}{*}{62.2} \\
\hline & Séptimo & 29.00 & 21.21 & & \\
\hline & Séptimo & 29.00 & 21.21 & \multirow[t]{2}{*}{.03} & \multirow[t]{2}{*}{51.2} \\
\hline & Primero & 28.17 & 25.51 & & \\
\hline & Séptimo & 29.00 & 21.21 & \multirow[t]{2}{*}{.15} & \multirow[t]{2}{*}{55.9} \\
\hline & Quinto & 25.66 & 23.05 & & \\
\hline \multirow{12}{*}{ Total } & Primero & 101.81 & 68.21 & \multirow[t]{2}{*}{.06} & \multirow[t]{2}{*}{52.4} \\
\hline & Quinto & 97.60 & 66.66 & & \\
\hline & Tercero & 121.72 & 83.61 & \multirow[t]{2}{*}{.26} & \multirow[t]{2}{*}{50.7} \\
\hline & Primero & 101.81 & 68.21 & & \\
\hline & Tercero & 121.72 & 83.61 & .31 & 62.2 \\
\hline & Quinto & 97.60 & 66.66 & & \\
\hline & Tercero & 121.72 & 83.61 & .06 & 52.4 \\
\hline & Séptimo & 116.63 & 62.28 & & \\
\hline & Séptimo & 116.63 & 62.28 & .22 & 58.7 \\
\hline & Primero & 101.81 & 68.21 & & \\
\hline & Séptimo & 116.63 & 62.28 & .28 & $61 \%$ \\
\hline & Quinto & 97.60 & 66.66 & & \\
\hline
\end{tabular}

tercer y primer semestres ( $d=.20)$, tercero con quinto semestres $(d=.30)$ y tercero con séptimo $(d=.31)$. En cuanto a ansiedad total, las magnitudes relevantes suceden entre tercer y primer semestres $(d=.26)$, tercero con quinto $(d=.31)$, entre séptimo con primero $(d=.22)$ y séptimo con quinto semestres $(d=.28)$.

Con los tamaños del efecto identificados es claro que los niveles de ansiedad se manifiestan de manera diferente entre los estudiantes según el semestre que se cursa, prevaleciendo primordialmente niveles más altos de ansiedad en los estudiantes de tercer semestre.

Si analizamos el porcentaje de estudiantes que presentaron ansiedad, observamos que es una cifra considerable: el 14.25\%; desglosando en sus diferentes 
modalidades, la ansiedad que más presentan es la fisiológica (19.27\%), siendo la motora y cognitiva casi iguales $(13.69 \%$ y $12.23 \%$, respectivamente). Esto nos indica que poco más del $12 \%$ están presentando conductas tales como preocupaciones, miedos, amenazas e inseguridades; que cerca del $20 \%$ de la población está somatizando esos sentimientos a través del aumento de la adrenalina, palpitaciones, taquicardia, escalofríos, transpiración, tensión muscular, respiración agitada y molestias físicas en general como mareos, dolores de estómago o de cabeza y poco más del $13 \%$ está presentado también inquietud, tics, temblores, reducción de la destreza motora y de ejecución de tareas complejas en general, así como conductas de evitación y escape de situaciones. Es interesante notar que estos resultados replican el orden y son muy cercanos a los porcentajes encontrados en la investigación de Aragón y cols. (2011), en cuanto a que la ansiedad más alta fue la fisiológica (15.5\%), seguida de la motora (15\%) y la cognitiva (12\%). Con respecto a las diferencias entre sexos, se encontraron diferencias significativas entre hombres y mujeres en ansiedad total, cognitiva y fisiológica, todas a favor de las mujeres. Estos resultados también concuerdan con los estudios anteriormente citados; en el de Aragón y cols. (2011) se encontró una diferencia significativa en ansiedad fisiológica también a favor de las mujeres; en el estudio de Sandin y cols. (2005) se encontraron puntuaciones significativamente más altas también en las mujeres en comparación con los hombres en ansiedad total, ansiedad somática y ansiedad cognitiva; y en la investigación de Sánchez y cols. (2006), donde no se encontraron diferencias significativas en ansiedad motora pero sí en ansiedad cognitiva, ansiedad fisiológica y ansiedad total, que fue exactamente lo que sucedió con nuestros resultados.

Al hacer un análisis mediante el tamaño del efecto ( $d$ de Cohen), se corrobora que el efecto más grande estuvo en aquellas diferencias que resultaron significativas, esto es, en ansiedad cognitiva, fisiológica y total, donde las mujeres obtuvieron significativamente puntuaciones más altas que los hombres, el efecto también fue mayor ya que la puntuación promedio de las mujeres rebasa al grupo de los hombres en un $64.06 \%$ en ansiedad cognitiva, en un $68.79 \%$ en ansiedad fisiológica y en un $61.41 \%$ en ansiedad total; en ansiedad motora, en que la diferencia no fue significativa, el tamaño del efecto fue de $51.99 \%$.

Encuanto a la comparación entre semestres, se encontró que la ansiedad cognitiva fue significativamente más alta entre los estudiantes de séptimo semestre comparados con los de quinto y primer semestres; seguramente estos resultados se deben a que dichos estudiantes se encuentran en el último año de la carrera y les produce preocupación y miedo el cumplimiento de las expectativas que tanto ellos como los demás esperan: terminar la licenciatura satisfactoriamente, titularse y empezar a ejercer la profesión. Asimismo, se encontró que los estudiantes de tercer semestre presentan significativamente más ansiedad cognitiva que los de quinto; esto se explica ya que dicha población comienza a atender odontológicamente a pacientes reales después de trabajar sólo con simuladores, además de tener la obligación de conseguir con sus propios recursos a los pacientes a atender, debido a que deben cumplir con un número determinado de tratamientos para acreditar la práctica clínica, y esto en ocasiones es difícil para ellos ya que algunos pacientes no se dejan atender por estudiantes que apenas inician con la clínica, lo que da como resultado que a veces los usuarios no regresan a terminar los tratamientos; aunado a esto, los profesores en este semestre les piden gran cantidad de trabajos.

\section{Conclusiones}

Podemos concluir que los estudiantes de la Carrera de Cirujano Dentista presentan en un porcentaje considerable ansiedad y que ésta se manifiesta en una quinta parte de ellos sobre todo a nivel fisiológico. Asimismo, las mujeres, al igual que en otros reportes de investigación, presentan significativamente más ansiedad que los hombres, tanto a nivel total como en sus manifestaciones fisiológicas y cognitivas, y que los semestres en los que más se está presentando este problema son en séptimo y tercero. Si bien no se encontraron diferencias significativas entre semestres en las otras modalidades de ansiedad, es interesante notar, considerando el tamaño del efecto, que las puntuaciones promedio de los alumnos de tercer semestre superan en al menos $61 \%$, en ansiedad fisiológica, a las puntuaciones de sus compañeros de primero y quinto semestres; y en ansiedad motora y total, a las puntuaciones de los de quinto y séptimo, es decir, independientemente de la significancia estadística, los alumnos del tercer semestre están presentando más ansiedad fisiológica, motora y total, que sus compañeros de los demás semestres.

Cabe mencionar que si bien los resultados de este estudio sólo corresponden a estudiantes de la FES Iztacala, y por otra parte, que quizá existan situaciones de ansiedad que no están representadas en el ISRA, estos datos pueden ayudar a crear programas tanto preventivos como de intervención con la finalidad 
de eliminar $o$ al menos aminorar el problema, ya que la ansiedad es una conducta que, de no tratarse oportunamente, puede interferir con el éxito escolar, así como ser en parte la causante de enfermedades más serias como colitis y úlceras, entre otras; ahí radica la importancia de este tipo de estudios en el campo de la Psicología de la Salud: crear programas de prevención e intervención.

Esta investigación en un futuro inmediato se centrará, una vez detectados los tipos de ansiedad que los estudiantes están presentando, en hacer un análisis de aquellas situaciones que están provocando dichas modalidades con el fin de iniciar programas de intervención.

Finalmente a lo antes mencionado y considerando que sucede en estudiantes de la Carrera de Cirujano Dentista, los resultados encontrados adquieren mayor relevancia por tratarse de profesionales del área de la salud, quienes necesariamente deben aplicar sus conocimientos, desarrollar habilidades y destrezas que le permitan ejercer su profesión con responsabilidad, ética y compromiso en beneficio de la comunidad.
Sánchez, L. M. P., Aparicio, G. M. E. y Dresch, V. (2006). Ansiedad, autoestima y satisfacción autopercibida como predictores de la salud: diferencias entre hombres y mujeres. Psicothema, 18(3), 584-590.

Sandin, B., Valiente, R. M., Chorot, P. y Santed, M. A. (2005). Propiedades psicométricas del índice de sensibilidad a la ansiedad. Psicothema, 17(3), 478-483.

Sue, D., Sue, D. W. y Sue, S. (2000). Understanding Abnormal Behavior. New York: Houghton Mifflin Company

Tobal, J. J. M. y Cano V. A. R. (1997).Inventario de Situaciones y Respuestas de Ansiedad. Madrid: TEA Ediciones, S. A.

Torres, Hernández-Pozo, Castillo, Coronado y Cerezo (2008). Relación entre promedio escolar y tres indicadores de ansiedad en estudiantes de medicina. Universitas Psychologica, 8(1), 69-86.

\section{Referencias}

Aragón, B. L. E. y Flores, T. M. A. (2014). Características de personalidad del estudiante de la carrera de Cirujano Dentista de la FES Iztacala, UNAM. Revista Odontológica Mexicana, 18(1), 19-26.

Aragón, B. L. E.; Contreras, G. O. y Trón A. R. (2011). Ansiedad y pensamiento constructivo en estudiantes universitarios. Journal of Behavior, Health and Social Issues, 3(1), 43-56.

García, G. E. y de la Peza, C. R. (2005). Relación de Variables Cognitivo- Emocionales y Rendimiento Académico. Revista Iberpsicología, 10(7).

Hernández-Pozo, Coronado, Araujo y Cerezo (2008). Desempeño académico de universitarios en relación con ansiedad escolar y autoevaluación. Acta Colombiana de Psicología, 11(1), 13-23.

González, C. A. L. (2014). Análisis complementarios a la significancia estadística: tamaño del efecto, significancia clínica y cambio clínico objetivo. Material Inédito, FES-Iztacala, UNAM. 4.0 Internacional, por lo que su contenido gráfico y escrito se puede compartir, copiar y redistribuir total o parcialmente sin necesidad de permiso expreso de sus autoras con la única condición de que no se puede usar con fines directamente comerciales y los términos legales de cualquier trabajo derivado deben ser los mismos que se expresan en la presente declaración. La única condición es que se cite la fuente con referencia a la Revista Digital Internaciona de Psicología y Ciencia Social y a sus autoras. 


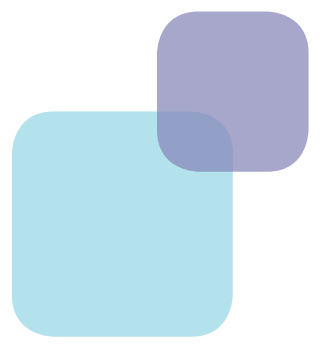

\section{MetA-ANÁLISIS DEL ARTículo}

A continuación se muestran los análisis de las puntuaciones otorgadas a este artículo por sus revisores 


\section{Dimensión Cuantitativa}

\section{Perfil de Evaluación entre Pares}
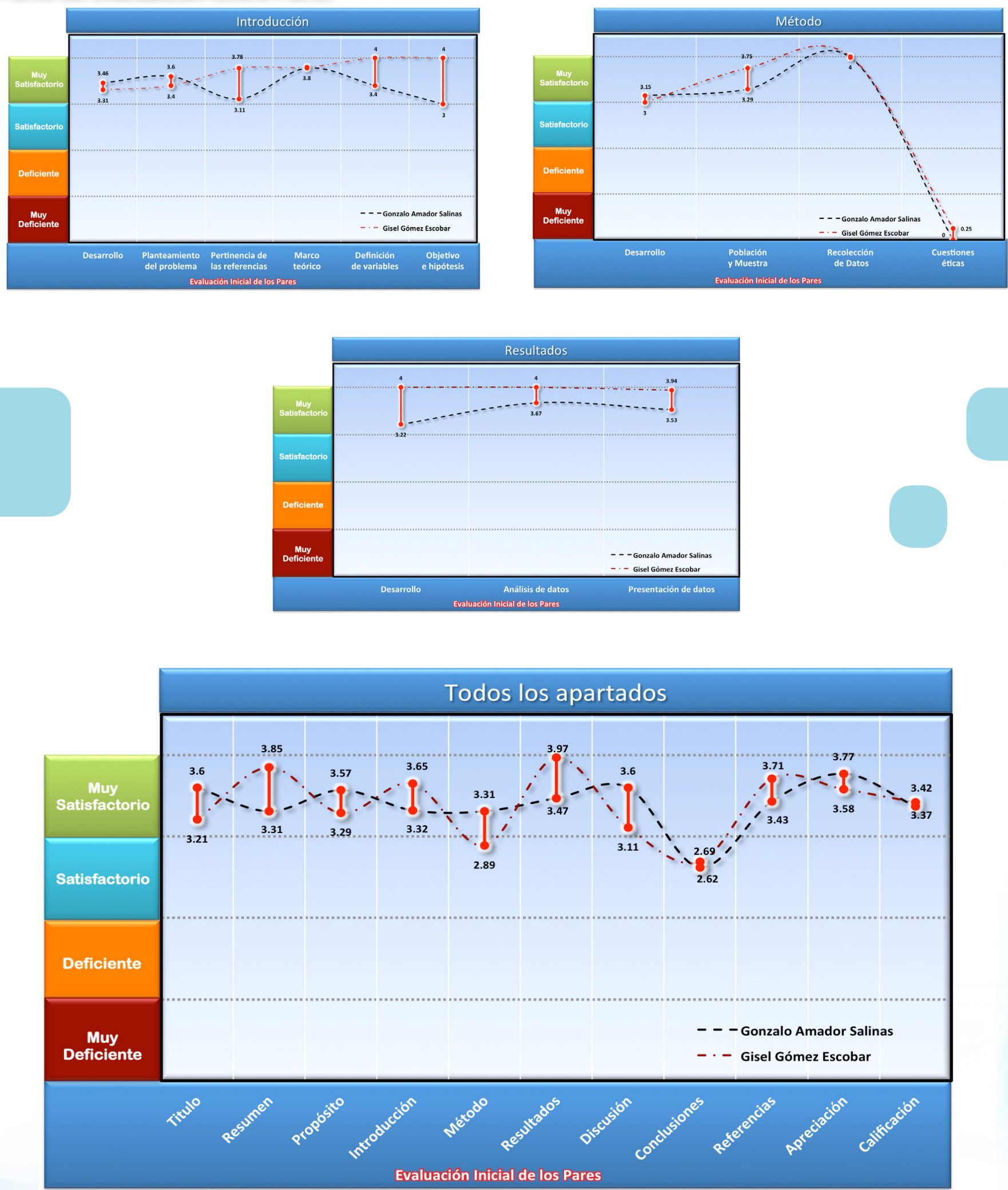


\section{Índice de Concordancia}

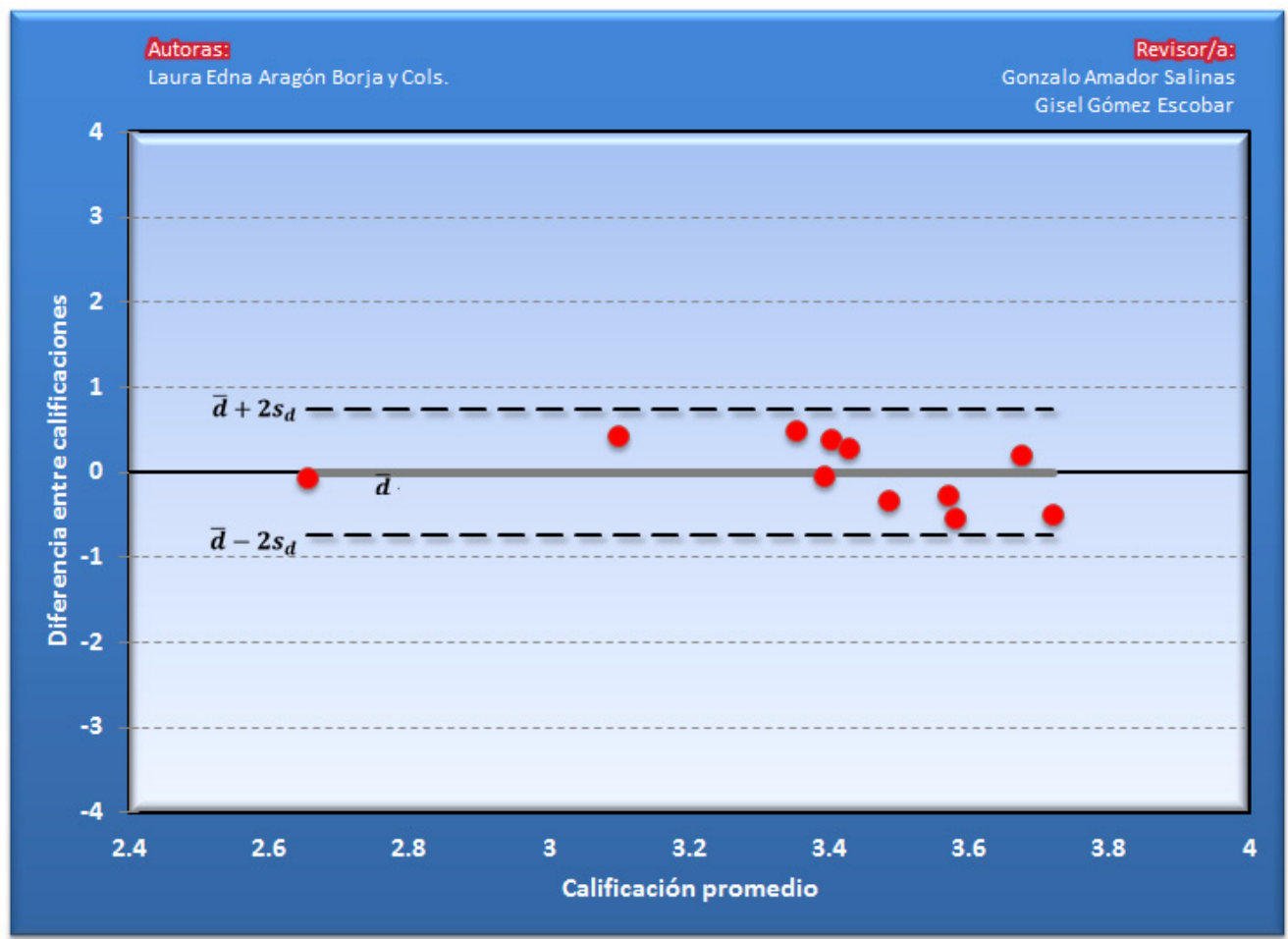

\section{Índice de Acuerdo}

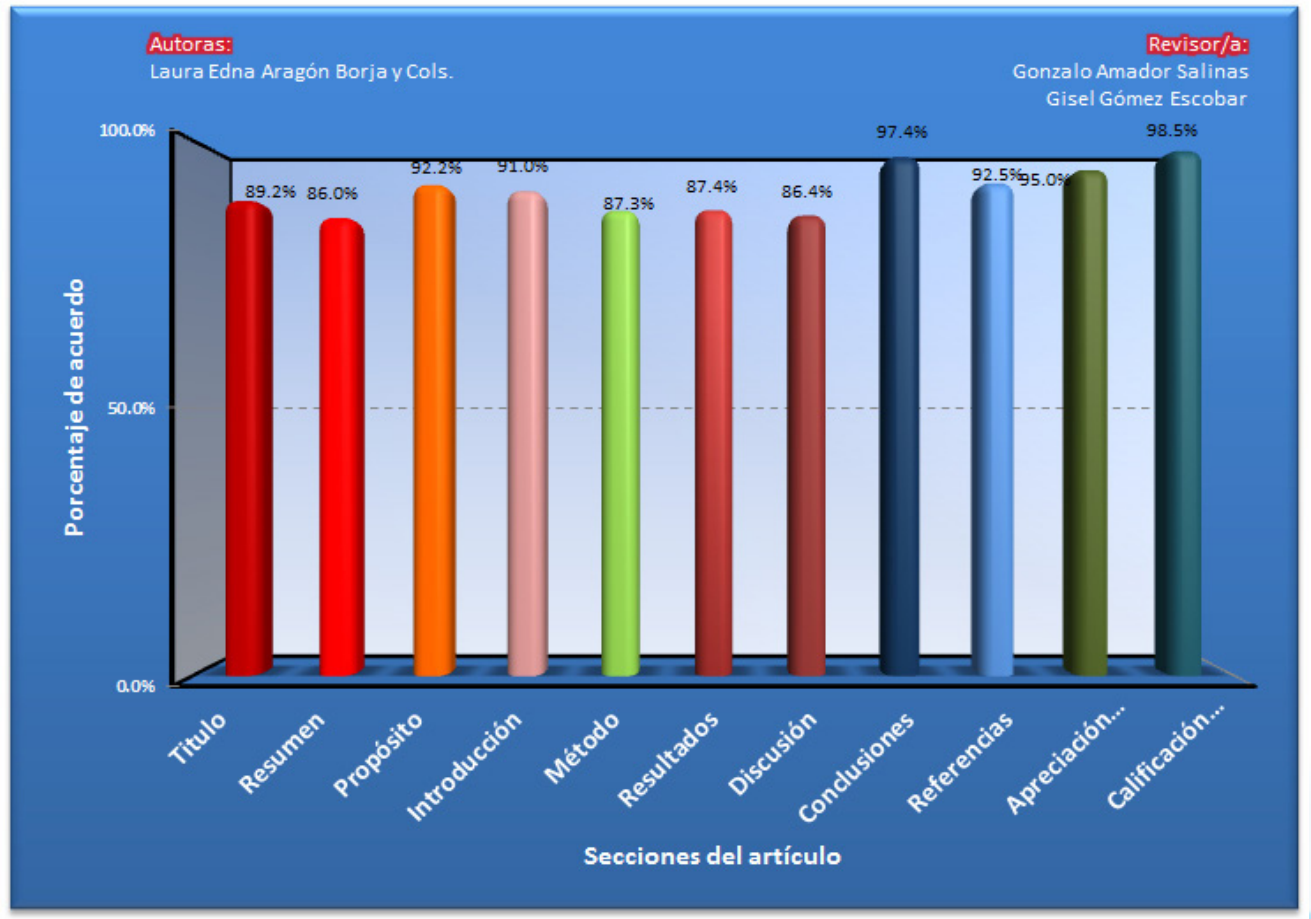




\section{Dimensión Cualitativa}

\begin{tabular}{|c|c|c|c|}
\hline \multicolumn{2}{c|}{ Revisor 1} & \multicolumn{1}{c}{ Revisor 2} \\
\hline José Gonzalo Amador Salinas & \multicolumn{1}{c}{ Gisel Gómez Escobar } \\
\hline Pues tendrían que colocar sus datos de contacto. & $\begin{array}{l}\text { Considero que podría quedar mejor así: Evaluación } \\
\text { de ansiedad en estudiantes de la carrera de Cirujano } \\
\text { Dentista. Especificando de inicio que es evaluación y no } \\
\text { tratamiento u otra; al final se puede omitir de dónde } \\
\text { son los estudiantes y mencionarlo en el método. }\end{array}$ \\
\hline
\end{tabular}

\section{RESUMEN}

Que incluyan la metodología y consideren el número de palabras.

En la traducción poner el nombre del inventario: Inventory of situations and anxiety responses.

\section{Próposito del Estudio}

Que incluyan las preguntas de investigación.

| Es congruente.

\section{INTRODUCCIÓN}

\begin{tabular}{l|l} 
Creo que la bibliografía puede ampliarse y actualizarse. & $\begin{array}{l}\text { Sólo comentar que la presentación de los estudios, } \\
\text { así como la justificación de por qué esas variables } \\
\text { demográficas, me parece que está bien justificada. }\end{array}$
\end{tabular}

\section{MÉTOdO}

\begin{tabular}{l|l} 
No está identificada la sección de materiales ni la la \\
estrategia de análisis de datos.
\end{tabular} $\begin{aligned} & \begin{array}{l}\text { Sólo hace falta especificar qué tipo de diseño emplearon } \\
\text { y detalles de la muestra sobre justificación del tamaño } \\
\text { y el tipo de asignación aleatoria empleada. }\end{array}\end{aligned}$

\section{Resultados}

$\begin{aligned} & \text { Me parece adecuada esta sección, solo revisar los } \\ & \text { dobles espacios. }\end{aligned}$

\section{Discusión}

Me parecería correcto que separaran la sección de discusión de la de conclusiones, y que en la discusión Incluir las limitaciones del estudio. compararan sus datos con más investigaciones.

\section{CONCLUSIONES}

Se propone que separen estas dos secciones, a manera $\mid$ Mencionar si hay campo de investigaciones futuras que mantener la claridad del texto. aborden el tema y por qué.

\section{REFERENCIAS}

Incluir mas bibliografía, y de preferencia actualizada. $\quad$ Bien.

continúa $\ldots$ 


\section{Impresión General del Manuscrito}

El artículo es por demás interesante, me parece que está bien estructurado y logra claridad en la exposición, tal vez es necesario ser más explícito en el problema detectado en la FESI, en la licenciatura de Odontología, el cual genera el estudio. En la sección de resultados, hay varios dobles espacios en el texto, por lo demás, es claro y concreto. Finalmente, en la sección de discusión y conclusiones, a mi parecer, podrían compararse estos resultados con otros similares en Instituciones de educación superior.

Mencionar cuál es la relevancia del estudio en el campo de la psicología y salud.

\section{COMENTARIO POR DICTAMEN}

Como lo he mencionado, en general está bien estructurado, es coherente y ágil en la redacción, las recomendaciones realizadas proponen la mejora del manuscrito, las cuales podríamos decir, son de forma y no de fondo.
Es claro y coherente el sustento teórico y empírico desde el principio hasta el final del estudio. El trabajo tiene un buen sustento metodológico y de aplicación de análisis estadísticos. 


\section{IR A LA Historia del Proceso EditORIAL}


